\title{
Elaboration of Reduced Graphene Oxide Electrodes Functionalized with rGO-ION Composite for Nitrite Detection in Water
}

\author{
A. Brahem ${ }^{1 *}$, A. Al-Hamry ${ }^{2}$, A. Reddy ${ }^{2}$, A. Bouhamed ${ }^{2}$, S. Ben Nasrallah ${ }^{1}$, L. G. Paterno ${ }^{3}$, M. Ben Ali1 ${ }^{1,4}$ and O. \\ Kanoun $^{2}$ \\ ${ }^{1}$ Electronic Department, University of Sousse, Higher Institute of Applied Science and Technology of Sousse, \\ Sousse, Tunisia \\ ${ }^{2}$ Technische Universität Chemnitz, Chair of Measurement and Sensor Technology, Chemnitz, Germany \\ ${ }^{3}$ Laboratório de Pesquisa em Polímeros e Nanomateriais, Instituto de Química, Universidade de Brasília, Brasilia, Brazil \\ ${ }^{4}$ NANOMISENE Lab, LR16CRMN01, Centre for Research on Microelectronics and Nanotechnology of Sousse, Technopole of \\ Sousse B.P. 334, Sahloul 4034, Sousse, Tunisia \\ *email: ammar.al-hamry@etit.tu-chemnitz.de
}

\begin{abstract}
In this paper, we propose a novel nitrite sensor based on reduced graphene oxide electrodes elaborated by light scribing. . First, graphene oxide was deposited on flexible substrate, which was previously plasma treated and wetted by a Poly(diallyldimethylammonium chloride) polymer solution. Then, in order to tune and enhance the sensitivity, the working electrode has been modified by layer-by-layer deposition of iron oxide-reduced graphene oxide nanocomposite (ION-rGO). The working electrode has been characterized at different levels of modification by scanning electron microscopy (SEM) and dispersive X-ray spectroscopy (EDX) showing a successful deposition and functionalization. Furthermore, electrochemical characterizations have been carried out by impedance spectroscopy and cyclic voltammetry $(\mathrm{CV})$ and quantitative measurements for nitrite detection by differential pulse voltammetry (DPV). The experimental results demonstrate a good electrochemical response for nitrite with a sensitivity of $0.314 \mu \mathrm{A} / \mathrm{mM}$. Several successive scans by $\mathrm{CV}$ at 50 $\mathrm{mV} / \mathrm{s}$ in $1 \mathrm{mM}$ nitrite solution show that the electrode remains stable and the sensitivity slightly reduces due to surface contaminations. The proposed fabrication procedure has a significantly better performance in comparison with some reported state-of-the art electrodes [12].
\end{abstract}

Keywords-component; Electrochemical sensors, Nitrite detection; Light scribe Graphene; Layer-by-Layer Deposition, reduced Graphene Oxide (rGO); Iron-oxide nanoparticles

\section{Introduction}

Over the last few years, in Germany due to excessive use of fertilizers, ground water is contaminated by nitrite in several regions, so that it has been condemned by the European court of justice for breach of EU law in this respect. Exceeding the allowed level of nitrite in drinking water causes several health problems, such as the "blue baby syndrome". Ingested nitrite can also react with amides and amines then forming potent carcinogens, such as nitrosamines [1-2]. The amount of nitrite in drinking water should therefore not exceed $3 \mathrm{mg} / \mathrm{L}$ referring to the World Health Organization (WHO) [3].

Hence, a reliable and accurate method for nitrite detection in water is important and recently received much attention. So far, many techniques have been adopted to detect nitrite, which involve tedious procedures and are time-consuming, such as chromatographic methods, ion spectrophotometry and chemiluminescence 4. Alternatively, electrochemical techniques are widely used because they provide highly selectivity, sensitivity and rapid nitrite determination compared with other traditional methods [5]. The electrochemical determination of nitrite is reported in literature either by oxidation or reduction. However, the oxidative nitrite determination is generally preferred because the nitrite reduction is basically known to suffer from interferences such as reduction of nitrate and molecular oxygen [6].

Recently, the applications of screen-printed electrodes (SPE) have significant advantages, such as simplicity, and low fabrication cost [7]. The whole electrode system, including working, reference and counter electrodes is printed on a substrate. Usually, for bare electrodes, the oxidation of nitrite occurs at high overpotential. Therefore, a modification of the screen printed electrodes is necessary in order to decrease the over potential and thereby to increase its sensitivity.

In this paper, we propose a novel nitrite sensors based on reduced graphene oxide electrodes produced by light scribing (LSG) [8-9]. To realize a high stability of the electrodes, graphene oxide was deposited on flexible substrate, which was plasma treated and wetted by a Poly(diallyldimethylammonium chloride) polymer solution. In order to tune and enhance the sensitivity, the working electrode has been modified by reduced graphene oxide- iron oxide nanoparticle (rGO-ION). The modification was made by layer-by-layer deposition of PDAC polymer and rGO-ION. The development of this modified electrode for nitrite detection is not expensive compared to conventional 
electrodes. This novel fabrication technique realizes lowcost sensors able to be used on-site.

\section{$2 \quad$ Material and methods}

\subsection{Chemicals}

The graphene oxide (GO) is commercially available in aqueous solution with a concentration of $4 \mathrm{~g} / \mathrm{L}$, prepared by modified Hummers method and it is obtained from Graphenea S.A, Spain. Iron oxide-reduced graphene oxide nanocomposite (ION-rGO) was synthesized as following; $200 \mathrm{~mL}$ of GO $0.1 \mathrm{~g} / \mathrm{L}$ with a $\mathrm{pH}$ of 11 was prepared. Under stirring, a solution of $0.5 \mathrm{~g}$ of $\mathrm{FeSO}_{4} .7 \mathrm{H}_{2} \mathrm{O}$ in $10 \mathrm{~mL}$ of deionized water was added to GO solution dropwise. The solution was stirred further for $30 \mathrm{~m}$ and the mixture was left decanting aided by a magnet. Cleaning steps were carried out by removing the supernatant and adding deionized water and decanting with the magnet. Finally, the particles were suspended NH3 solution ( $\mathrm{pH} 9$ ) using the horn sonicator and then centrifugated.

In this study, all reagents used were of analytical grade and distilled water was used throughout. Sodium nitrite was purchased from Aldrich. Freshly made 0.1 M phosphate buffer solution (PBS) in different pHs, consisting of sodium phosphate dibasic (Na2HPO4) and sodium phosphate monobasic (NaH2PO4) was used during experimental testes as the supporting electrolyte.

\subsection{Preparation of the electrodes}

The preparation of LSG was done by using a commercially available laser source (LightScribe DVD drive with $788 \mathrm{~nm}$ ) controlled by means of a computer software (Nero Cover Design) to reduce the graphene oxide (GO). For that, GO in an aqueous solution of 0.3 wt. \% was casted directly onto polyethylene terephthalate (PET) substrate which was plasma treated. The substrate was already glued to a DVD disc by adhesive spray. GO takes $24 \mathrm{~h}$ to dry under ambient conditions. Then, the obtained film was reduced by light scribing method. More than 20 electrodes can be produced on a single DVD disc. The electrodes were prepared by pasting $\mathrm{Ag} / \mathrm{AgCl}$ to the reference electrode (RE). Working electrodes (WE) were functionalized with the ION-rGO nanocomposite, while counter electrodes (CE) were kept unchanged i.e. only reduced graphene oxide (rGO). Finally, a passivation layer was applied to obtain final electrode as shown in Fig 1a.

rGO working electrodes were functionalized with Layerby-layer technique using tow oppositely charged materials The first step was drop casting a layer of PDAC polymer on the working electrode WE and washed by deionized water in order to remove unbound material. The same procedure is repeated to deposit ION-rGO nanocomposite as shown in the figure 1b. This process forms one bilayer. Finally, the functionalized electrode was dried with nitrogen gas and it is ready for the electrochemical testes.

\subsection{Characterization and Measurement Setup}

The examination of the electrode morphology was carried out by a scanning electron microscopy (SEM) equipped with an energy dispersive X-ray spectroscopy (EDX). For the electrochemical experiments, PalmSens 4 (PalmSens $\mathrm{BV}$ ) was used to perform cyclic voltammetry (CV) and differential pulse voltammetry (DPV) measurements.

a)

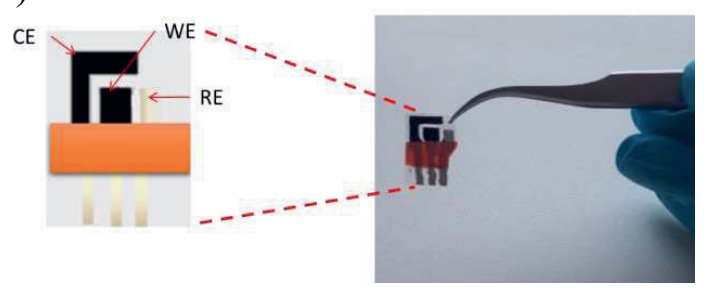

b)

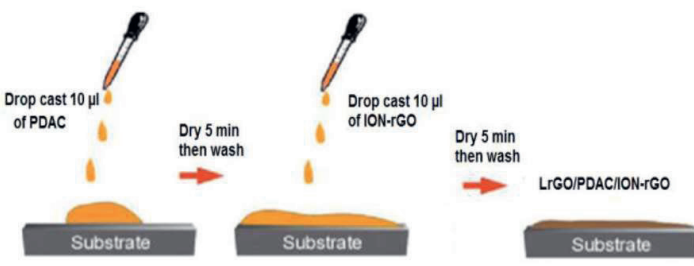

Fig1. a) LSG fabricated electrode configuration and b) Graphical illustration of the electrode functionalization

\section{Results and Discussion}

\subsection{Surface Characterization electrodes}

The morphology of electrodes after each preparation step can be clearly seen from the SEM images displayed in in Fig2. In Fig. 2a, the initial GO cast film shows a homogeneous film. After laser-scribing, when GO is photothermally reduced to $\mathrm{rGO}$, the surface becomes rougher. This morphological transformation may be a consequence of a chemical bonds breaking during the reduction process. The lattice defects during the surface reconstruction after removing hydroxyl and epoxy groups may also contributed to change the morphology. The formation of rGO was further confirmed by EDX, which is performed using the same samples. EDX results show that carbon to oxygen relative concentration increase from GO to rGO samples, proving the reduction efficacy of the laser scribing process. . Finally, the last step of modification with PDAC/ION-rGO nanocomposite film is seen in Fig.2c. . It is possible to detect rGO sheets decorate with nearly spherical nanoparticles, which are the ION. EDX measurements detected the presence of iron and confirm the successful functionalization of the working electrode. 

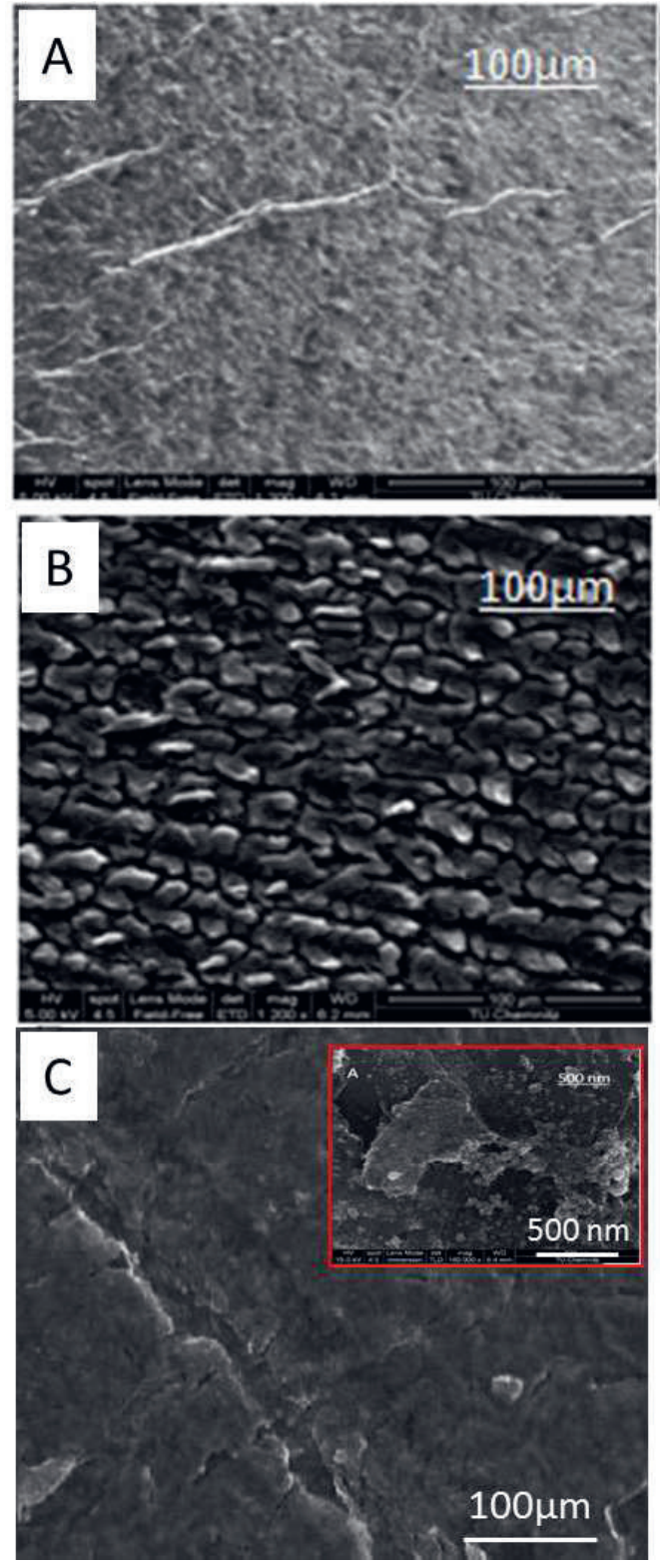

Fig 2. SEM images of (a) graphene oxide on top of the PET substrate, (b) rGO obtained after laser scribing, and (c) PDAC/ION-rGO nancomposite film on top of the laser scribed rGO electrode, Inset: image with higher magnification with noticeable ION nanparticles

\subsection{Electrochemical behavior}

First of all ,a test of the electrode without target ion with a solution composed of $0.1 \mathrm{M}$ of $\mathrm{KCl}$ and $10^{-3} \mathrm{M}$ ferri/ferrocyanide $[\mathrm{Fe}(\mathrm{CN}) 6]^{3-4-}$ was performed. For both bare and modified (PDAC/ION-rGO) electrodes we tested by $\mathrm{CV}$, Fig.3. For modified surfaces, the $[\mathrm{Fe}(\mathrm{CN}) 6]^{3-/ 4-}$ redox couple provides a good electrochemical probe to study, because its oxidation and reduction proceed via a simple oneelectron-transfer process. This chemical reaction (1) is the base of this redox-couple's electrochemistry:

$$
\begin{gathered}
{\left[\mathrm{Fe}(\mathrm{CN})_{6}\right]^{3-}+\left[\mathrm{Fe}(\mathrm{CN})_{6}\right]^{3-} \mathrm{e}^{-} \leftrightarrow} \\
{\left[\mathrm{Fe}(\mathrm{CN})_{6}\right]^{4-}}
\end{gathered}
$$

An improvement between bare and modified electrodes in the electrochemical response can be seen in Fig. 3 by the increase of the ferri/ferrocyanide oxidation and reduction peak currents after electrode modification with the PDAC/ION-rGO film. This improvement is ascribed to the increase of the surface area provided by the electrode modification.

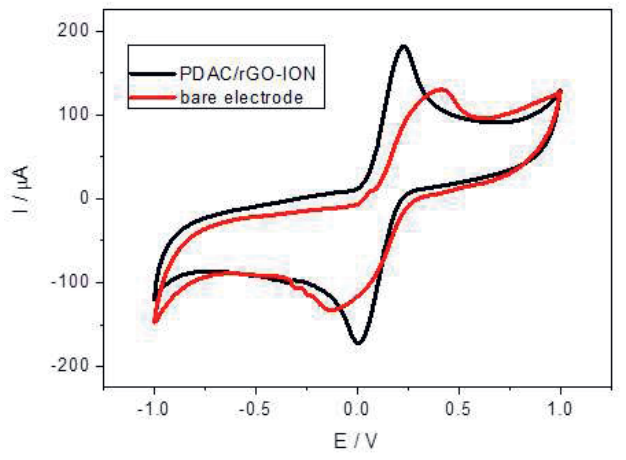

Fig.3.Cyclic voltammograms of Ferri-Ferrocyanide solution $10^{-3} \mathrm{M}$ of bare and modified electrode in 25 $\mathrm{mV} / \mathrm{s}$

$\mathrm{CV}$ was also performed at different scan rates $(25 \mathrm{mV} / \mathrm{s}, 50$ $\mathrm{mV} / \mathrm{s}, 100 \mathrm{mV} / \mathrm{s}, 150 \mathrm{mV} / \mathrm{s}, 200 \mathrm{mV} / \mathrm{s}$. It was found a linear relationship between anodic/cathodic peak currents $\left(i_{p}\right)$ and the square-root of the scan rate $\left(v^{1 / 2}\right)$, shown in Fig.4.

The electro-active surface area of the electrodes was calculated by using the Randles-Sevcik equation. This equation predicts that the current peak should be proportional to the square root of the scan rate when voltammograms are taken at different scan rates. At $25{ }^{\circ} \mathrm{C}$, the Randles-Sevcik equation can be reduced to the following equation (2) [11]:

$$
i_{p}=2,6910^{5} n^{\frac{3}{2}} A D^{\frac{1}{2}} C v^{\frac{1}{2}}
$$

in which $\mathrm{n}$ is the number of electrons appearing in half reaction for the redox couple ( $\mathrm{n}=1$ in our case), $\mathrm{A}$ is the active surface of the electrode $\left(\mathrm{cm}^{2}\right), \mathrm{C}$ represents the concentration of the electroactive species $\left(\mathrm{mol} \mathrm{cm}^{-3}\right), v$ is the speed at which the potential is swept $(\mathrm{V} / \mathrm{s})$, and $\mathrm{D}$ is the diffusion coefficient of the analytes $\left(\mathrm{cm}^{2} \mathrm{~s}^{-1}\right)$. From this equation, we can directly calculate the electroactive area for bare and modified electrodes.

Before the modification $\mathrm{A}$ is $0.2 \mathrm{~cm}^{2}$, and it increases to $0.29 \mathrm{~cm}^{2}$ after functionalization with the layer-by-layer assembled PDAC/ION-rGO film.

To choose the proper $\mathrm{pH}$ value at which the best detection of nitrite takes place, $\mathrm{CV}$ was performed by changing the $\mathrm{pH}$ value of the PBS solution: 3.5, 4.5, 5.7, 6.0, 6.5 and 7.4 as shown in in Fig.5a and b. The nitrite concentration is fixed at $1 \mathrm{mM}$ as well as scan rate used is fixed at $50 \mathrm{mV} / \mathrm{s}$. It is found that oxidation current of nitrite ions for the modified electrodes has a better peak achieved at $\mathrm{pH}=5.7$. 


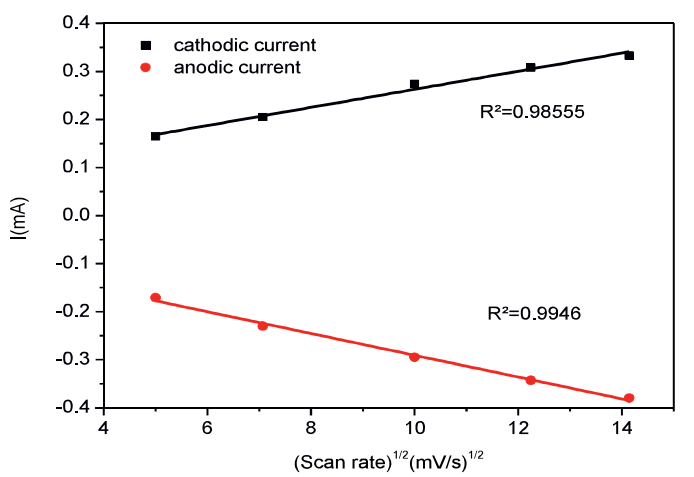

Fig. 4 Anodic and cathodic current of nitrite versus Square root of scan rate Vs

The sensor response to nitrite detection was tested by differential pulse voltammetry (DPV). The DPV curves of the modified electrode following the change in nitrite ion concentration were measured and shown in Fig. 6. The peak oxidation current increases with increasing the nitrite concentrations. This increase is almost linear.

a)

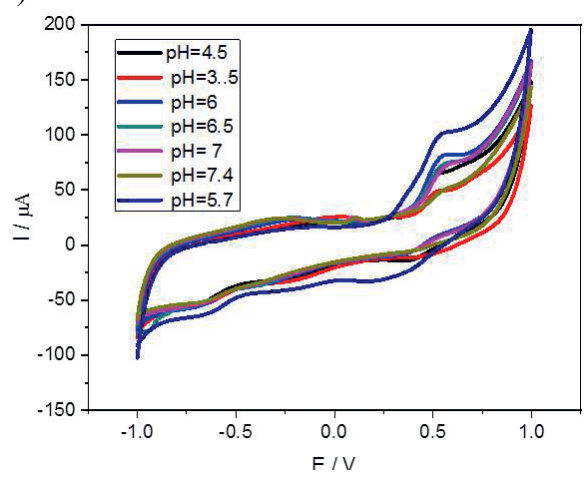

b)

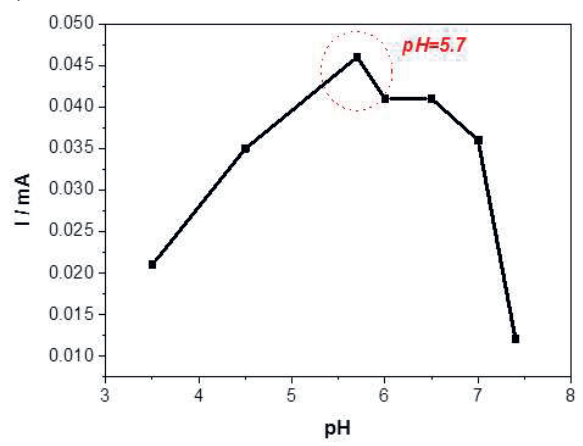

Fig. 5. (a) Cyclic voltammograms of detection of nitrite in different $\mathrm{pHs}$ and (b) Variation of nitrite oxidation peak current value with the $\mathrm{pH}$. All measurements performed with the LSG PDAC/ION-rGO electrode.

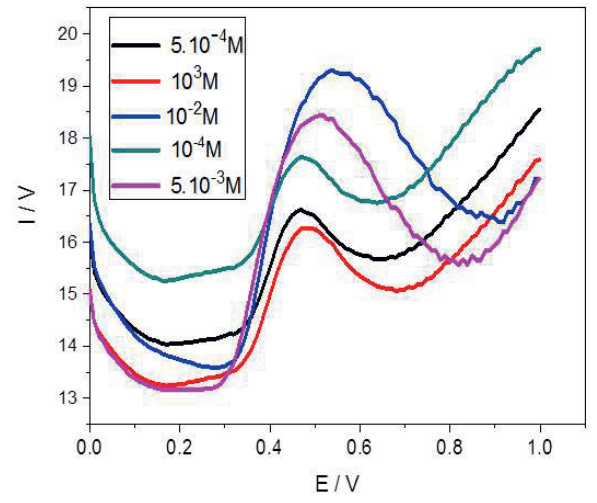

Fig. 6: DPV of modified electrode with RGOIONs/PDAC for different nitrite concentrations

Fig. 7 illustrates the calibration curve describing the variation of currents as a function of increasing nitrite concentrations. We find that the method has a sensitivity of 0.314 $\mu \mathrm{A} / \mathrm{mA}$ with $\left(\mathrm{R}^{2}=0.93\right)$.

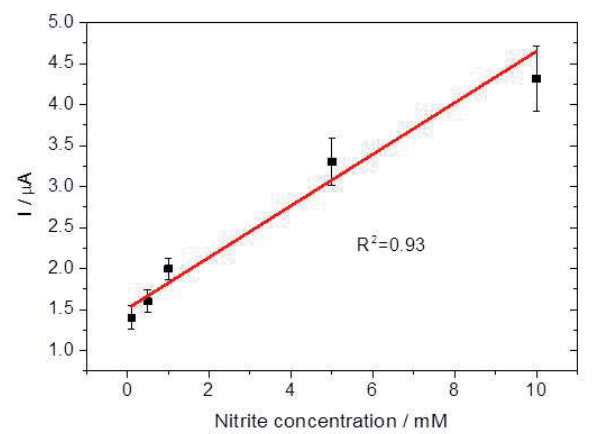

Fig.7 Nitrite calibration curve

Moreover, the reproducibility was studied as an important parameter of sensor characterization. The reproducibility was investigated from the current and potential response from $\mathrm{CV}$ using four electrodes independently prepared in the same way as seen in Fig. 8. Furthermore, the electrodes show stability of the oxidation peak current even after tens of cycles. Several successive scans by cyclic voltammetry of with a rate of $50 \mathrm{mV} / \mathrm{s}$ in $1 \mathrm{mM}$ nitrite solution show that the electrode remains stable and using plasma and PDAC wetting make the electrode more stable. 


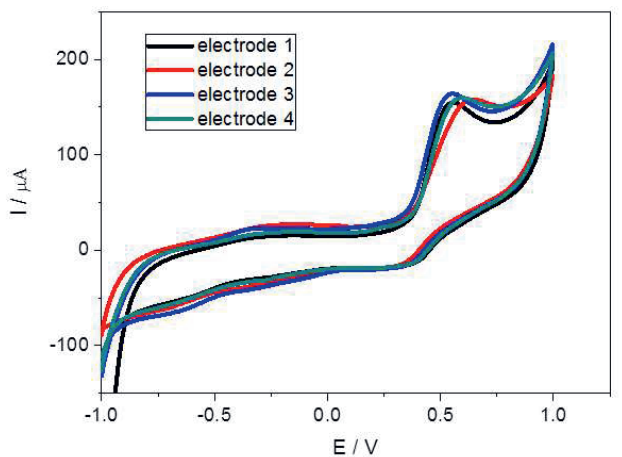

Fig. 8 Cyclic voltammograms of nitrite registered with four LSG PDAC/ION-rGO electrodes produced in different batches.

\section{Conclusion}

In this work, sensitive electrodes for the detection of nitrite in water were developed using PDAC/ION-rGO modified laser-scribed reduced graphene oxide electrode. The electrodes' modification was performed by layer-by-layer deposition technique. The sensor has a sensitivity of 0.314 $\mu \mathrm{A} / \mathrm{mM}$, which is better than state of the art sensors [12].

This study combines the use of nanomaterials and the realization of low-cost electrodes, which can be disposable, in the detection of nitrite in water. This can be an alternative for the detection and sensing of many other substances. The realized sensor has a fast response and is economically feasible.

\section{Acknowledgement}

This work was carried out with the support of the Nitramon project (project no. 100339427) funded by the Sächsische Aufbaubank (SAB) and the European Social Fund (ESF) and the project Electrochemical Sensors for Environmental Applications (ESEA) (project no. 249305) funded by DAAD. Authors acknowledge the support of Aleksandr Oshchepkov (within the project Nitramon) from Technische Universität Chemnitz, group of supramolecular chemistry, chemical department, for solutions preparation and discussions. A. A.-H. and O. K. acknowledge the financial support within the project InnoTeam-SimplySafe (contr. nr.100331073) funded by the Sächsische Aufbaubank (SAB) and the European Social Fund (ESF). L.G. P. and O. K. acknowledge the Alexander Von Humboldt Foundation.

\section{Literature}

1. Bryan, N.S., 2006. Nitrite in nitric oxide biology: Cause or consequence?: A systems-based review. Free Radical Biology and Medicine, 41(5), pp.691-701.
2. Chen, K.K. and Rose, C.L., 1952. Nitrite and thiosulfate therapy in cyanide poisoning. Journal of the American Medical Association, 149(2), pp.113-119.

3. Gorchev, H.G. and Ozolins, G., 1984. WHO guidelines for drinking-water quality. WHO chronicle.

4. Wang, Q.H., Yu, L.J., Liu, Y., Lin, L., Lu, R.G., Zhu, J.P., He, L. and Lu, Z.L., 2017. Methods for the detection and determination of nitrite and nitrate: A review. Talanta, 165, pp.709-720.

5. Kalimuthu, P. and John, S.A., 2009. Highly sensitive and selective amperometric determination of nitrite using electropolymerized film of functionalized thiadiazole modified glassy carbon electrode. Electrochemistry Communications, 11(5), pp.1065-1068.

6. Caro, C.A., Bedioui, F. and Zagal, J.H., 2002. Electrocatalytic oxidation of nitrite on a vitreous carbon electrode modified with cobalt phthalocyanine. Electrochimica Acta, 47(9), pp.1489-1494.

7. O'Halloran, M.P.; Pravda, M.; Guilbault, G.G. Prussian Blue bulk modified screen-printed electrodes for $\mathrm{H} 2 \mathrm{O} 2$ detection and for biosensors. Talanta 2001, 55, 605-611.

8. Rodriguez, R. R. et al: "Reduced Graphene Oxide Nanostructures by Light: Going Beyond the Diffraction Limit" 2018 J. Phys.: Conf. Ser.1092 012124

9. Al-Hamry, A. et. al.: "Tuning the reduction and conductivity of solution-processed graphene oxide by intense pulsed light", Carbon, Vol. 102, June 2016, Pages 236-244

10. Cheng, K.H., Cheng, C.H., Chao, A.K.A. and Lo, D.K.C., 2015, April. LASER light scribe and microwave annealing reduction of graphene oxide for supercapacitor applications. In 10th IEEE International Conference on Nano/Micro Engineered and Molecular Systems (pp. 380-383). IEEE.

11. M. E. G. Lyons and G. P. Keeley, "The Redox Behaviour of Randomly Dispersed Single walled carbon nanotubes both in the absence and in the presence of adsorbed glucose oxidase," Sensors, vol. 6, no. 12, pp. 1791-1826, 2006.

12. Amira Ghezal et. al.: "Electrochemical detection of nitrite using screen printed graphite electrode modified by ION/rGO", accepted for publication in the 16th International Conference on Systems, Signals and Devices 2019, 21-24 March 2019, Istanbul, Turkey 